\title{
VINCENT BRUNEAU
}

\section{Asymptotique de la phase de diffusion à haute énergie pour l'opérateur de Dirac}

\author{
Annales de la faculté des sciences de Toulouse $6^{e}$ série, tome $7, \mathrm{n}^{\circ} 2$ \\ (1998), p. 207-231 \\ <http://www.numdam.org/item?id=AFST_1998_6_7_2_207_0>
}

(C) Université Paul Sabatier, 1998, tous droits réservés.

L'accès aux archives de la revue «Annales de la faculté des sciences de Toulouse » (http://picard.ups-tlse.fr/ annales/) implique l'accord avec les conditions générales d'utilisation (http://www.numdam.org/conditions). Toute utilisation commerciale ou impression systématique est constitutive d'une infraction pénale. Toute copie ou impression de ce fichier doit contenir la présente mention de copyright.

\section{Numdam}

Article numérisé dans le cadre du programme Numérisation de documents anciens mathématiques http://www.numdam.org/ 


\title{
Asymptotique de \\ la phase de diffusion à haute énergie pour l'opérateur de Dirac ${ }^{(*)}$
}

\author{
VinCENT BRUNEAU ${ }^{(1)}$
}

RÉsuMÉ. - Nous étudions le comportement asymptotique, quand $\lambda \rightarrow$ $\pm \infty$, de la phase de diffusion (ou fonction spectrale de perturbation $s_{H}(\lambda)$, pour $H$, l'opérateur de Dirac perturbé par un potentiel à court. portée. Une formule de type Weyl est établie pour un potentiel électrc magnétique. Si le potentiel est uniquement magnétique, le développemen asymptotique complet de $s_{H}$ se déduit de celui de $s_{H^{2}}$, la phase de dif fusion pour $H^{2}$ (une perturbation du laplacien). Ce lien entre $s_{H}$ et $s_{H}$ : provient des propriétés de supersymétrie que nous exploitons aussi pour aborder l'étude de l'opérateur de Hodge-Dirac dont le carré est l'opérateur de Hodge-Laplace.

ABstract. - We examine the scattering phase related to electromagnetic perturbations of the Dirac operator. For a short-range electromagnetic potential, we prove a Weyl type formula. For a magnetic potential, we obtain a full asymptotic expansion using a supersymmetry property. Using the same arguments we study the scattering phase for the HodgeDirac operator.

\section{Introduction}

Nous considérons l'opérateur de Dirac dans $\mathbb{R}^{3}$ avec champ électromagnétique :

$$
H=c \sum_{j=1}^{3} \alpha_{j}\left(\hbar \mathrm{D}_{j}-A_{j}(x)\right)+\beta m c^{2}+V(x), \quad \mathrm{D}_{j} \stackrel{\text { def }}{=}-i \partial_{x_{j}}
$$

(*) Reçu le 28 mai 1996, accepté le 9 septembre 1996

(1) Mathématiques appliquées de Bordeaux, Université Bordeaux 1, 351 cours de la Libération, F-33405 Talence (France)

E-mail : vbruneau@math.u-bordeaux.fr 


\section{Vincent Bruneau}

où $\left\{\alpha_{j}\right\}_{j=1}^{3}$ et $\beta$ sont les matrices $4 \times 4$ de Dirac, $A=\left(A_{1}, A_{2}, A_{3}\right)$ est le potentiel magnétique et

$$
V=\left(\begin{array}{cc}
V_{+} 1_{2} & 0 \\
0 & V_{-} 1_{2}
\end{array}\right)
$$

avec $V_{ \pm}$scalaire $\left(1_{2}\right.$ est la matrice identité de $\left.\mathbb{C}^{2}\right)$. Les constantes physiques $c$ (vitesse de la lumière), $m$ (masse) et $\hbar$ (constante de Planck) sont fixées.

Nous supposons que les potentiels sont $C^{\infty}$ et qu'il existe $\delta>0$ tel que :

$\forall \alpha \in \mathbb{N}^{3}, \quad\left|\partial_{x}^{\alpha} A(x)\right|+\left|\partial_{x}^{\alpha} V(x)\right|=\mathrm{O}\left(\langle x\rangle^{-\delta-|\alpha|}\right), \quad\langle x\rangle \stackrel{\text { def }}{=}\left(1+|x|^{2}\right)^{1 / 2}$.

On note encore $H$, l'extension : utoadjointe sur $L^{2}\left(\mathbb{R}^{3}\right)^{4}$, de domaine $H^{1}\left(\mathbb{R}^{3}\right)^{4}$.

$H$ est une perturbation de l'op, ateur de Dirac libre :

$$
H_{0}=c \sum_{j=1}^{3} \alpha_{j} \hbar \mathrm{D}_{j}+m c^{2} \beta
$$

dont le spectre est purement absolument continu :

$$
\left.\left.\sigma\left(H_{0}\right)=\sigma_{c}\left(H_{0}\right)=\sigma_{a c}\left(H_{0}\right)=\right]-\infty,-m c^{2}\right] \cup\left[m c^{2},+\infty[.\right.
$$

Pour $\delta>1$, on a les propriétés suivantes ([19], [21]) :

(i) les opérateurs d'onde associés au couple $\left(H, H_{0}\right)$ existent et sont complets;

(ii) le spectre essentiel de $H$ est égal à $\left.]-\infty,-m c^{2}\right] \cup\left[m c^{2},+\infty[\right.$;

(iii) le spectre singulièrement continu de $H$ est vide;

(iv) les valeurs propres de $H$ sont localisées dans $\left[-m c^{2}, m c^{2}\right]$.

Dans cet article, nous étudions les propriétés asymptotiques du spectre continu de $H$, à l'aide de la phase de diffusion, définie comme suit, pour la paire $\left(H, H_{0}\right)$.

D'après (i), l'opérateur de diffusion $S$ est défini et unitaire. L'opérateur $S$ commute avec $H_{0}$, sa représentation en énergie $S(\lambda)$ définit la matrice de diffusion, pour $\left.\lambda \in]-\infty,-m c^{2}\right] \cup\left[m c^{2},+\infty[\right.$ [. L'opérateur $S(\lambda)$ est unitaire sur $L^{2}\left(S^{2}\right)^{4}\left(S^{2}\right.$ est la sphère unité de $\left.\mathbb{R}^{3}\right)$. 
Asymptotique de la phase de diffusion à haute énergie pour l'opérateur de Dirac

Pour $\delta>3, S(\lambda)-1$ est un opérateur de classe trace dans $L^{2}\left(S^{2}\right)^{4}$, et l'égalité suivante définit la phase de diffusion $s(\lambda)$ (modulo $\mathbb{Z}$ ) :

$$
\operatorname{det}(S(\lambda))=\exp (-2 i \pi s(\lambda))
$$

D'après la théorie de Birman-Krein [2], on peut définir la phase de diffusion globalement sur $\mathbb{R}$, à une constante près, par la formule de Krein :

$$
\operatorname{Tr}\left(f(H)-f\left(H_{0}\right)\right)=\int_{\mathbb{R}} s(\lambda) f^{\prime}(\lambda) \mathrm{d} \lambda, \quad \forall f \in \mathcal{S}(\mathbb{R}) .
$$

Celle-ci étend, au spectre continu, la notion de fonction de répartition des valeurs propres.

L'hypothèse $\left(\mathrm{H}_{\delta}\right)$ entraine que $s \in C^{\infty}(]-\infty,-m c^{2}[\cup] m c^{2},+\infty[)$. Ceci se démontre comme pour l'opérateur de Schrödinger (cf. [15, corollaire 5.8]) à partir de la représentation stationnaire de la matrice de diffusion donnée par E. Balslev et B. Helffer [1].

Dans cet article, on fixe $m=c=\hbar=1$, et on étudie le comportement asymptotique de $s(\lambda)$ quand $\lambda \rightarrow \pm \infty$. Cela revient à étudier celui de

$$
s_{h}(\mu) \stackrel{\text { def }}{=} s\left(\frac{\mu}{h}\right)
$$

pour $\pm \mu>0$ fixé quand $h \searrow 0$. À la section 3, on commence par faire une étude au sens des distributions. D'après la formule de Birman-Krein (1), cela consiste à étudier le comportement asymptotique quand $h \searrow 0$ de $\operatorname{Tr}\left(f(h H)-f\left(h H_{0}\right)\right)$ pour $f \in \mathcal{S}(\mathbb{R})$, nulle au voisinage de 0.

Ensuite, on considère $s(\lambda)$ non plus comme distribution, mais comme fonction. L'existence d'une asymptotique du type Weyl est établie à la section 4. Celle-ci repose sur la construction d'un opérateur Fourier intégral (O.F.I.) qui approche le propagateur à temps petit (sect. 5).

Enfin, nous donnons un développement asymptotique complet de $s(\lambda)$ dans le cas particulier $V=0$. Celui-ci se déduit directement de l'étude réalisée par D. Robert [15] pour des opérateurs de type Schrödinger, en utilisant les propriétés de supersymétrie que possède l'opérateur de Dirac avec champ magnétique. Nous indiquons de plus comment ces propriétés permettent aussi d'étudier l'opérateur de Hodge-Dirac grâce à un théorème d'indice relatif [3].

Les principaux résultats présentés ici étaient annoncés dans [5] et résument essentiellement les chapitres 2 et 3 de [4]. 


\section{2 . Énoncés des résultats}

Nous utiliserons librement la théorie des opérateurs pseudo-différentiels. Une quantification bien adaptée au calcul de traces est la quantification de Weyl. À un symbole $a$ et un réel $h>0$, elle associe un opérateur $\operatorname{Op}_{h}^{\omega}(a)$ défini pour $u \in \mathcal{S}(\mathbb{R})$, par :

$$
\left(\mathrm{Op}_{h}^{\omega}(a) u\right)(x)=(2 \pi)^{-n} \iint e^{i(x-y, \xi)} a\left(\frac{x+y}{2}, h \xi\right) u(y) \mathrm{d} y \mathrm{~d} \xi
$$

Pour plus de détails concernant les classes de symboles, nous renvoyons au livre [13] et $[4, \S 1.8]$.

Dans la suite, $a_{0}(\xi)=\alpha \cdot \xi$ désigne le symbole principal de $H$, et $a_{1}(x)=-\alpha \cdot A(x)+\beta m c^{2}+V(x)$ le symbole sous-principal ( $a_{0}$ et $a_{1}$ sont des matrices). L'opérateur $h H$ est $h$-admissible:

$$
h H=\mathrm{Op}_{h}^{\omega}\left(a_{0}\right)+h \mathrm{Op} \mathrm{p}_{h}^{\omega}\left(a_{1}\right) .
$$

THÉORÈME 2.1. - (Asymptotique au sens faible) Soit $\phi \in \mathcal{S}(\mathbb{R})$ nulle au voisinage de 0 . Sous l'hypothèse $\left(H_{\delta}\right)$, on a

i) l'opérateur $\phi(h H)$ est $h$-admissible de symbole principal $\phi\left(a_{0}\right)$, et de symbole sous-principal

$$
\begin{gathered}
a_{\phi, 1}=a_{1, D} \phi^{\prime}\left(a_{0}\right)+\frac{1}{2} a_{1, M}\left(a_{0}\right)^{-1}\left(\phi\left(a_{0}\right)-\phi\left(-a_{0}\right)\right), \\
\text { où } a_{1, D}=P^{+} a_{1} P^{+}+P^{-} a_{1} P^{-} \text {et } a_{1, M}=P^{+} a_{1} P^{-}+P^{-} a_{1} P^{+}, \\
P^{ \pm} \stackrel{\text { def }}{=} \frac{1}{2}\left(1 \pm \frac{\alpha \cdot \xi}{|\xi|}\right)
\end{gathered}
$$

étant la projection orthogonale sur le sous-espace propre associé à la valeur propre $\pm|\xi|$ de $\alpha \cdot \xi$;

ii) si de plus $\delta>3$, alors on a le développement asymptotique suivant:

$$
\operatorname{Tr}\left(\phi(h H)-\phi\left(h H_{0}\right)\right) \asymp(2 \pi h)^{-3} \sum_{j \geq 1} \gamma_{j}(\phi) h^{j} \quad \text { lorsque } h \searrow 0,
$$

les coefficients $\gamma_{j}(\phi)$ sont des distributions par rapport $\grave{a} \phi$, calculables en fonction de $A, V$ et leurs dérivées. 
Asymptotique de la phase de diffusion à haute énergie pour l'opérateur de Dirac

Notons que le phénomène d'interaction entre les énergies positives et négatives, propre à la théorie de Dirac, apparait dans le symbole sousprincipal avec $a_{1, M}$.

THÉORÈME 2.2.- (Formule de type Weyl) On suppose $\left(H_{\delta}\right)$, avec $\delta>3$. On a alors, une asymptotique du type Weyl pour $s(\lambda)$ :

$$
s(\lambda)=(2 \pi)^{-3} \alpha_{1} \lambda^{2}+\mathrm{O}(\lambda), \quad \lambda \rightarrow \pm \infty .
$$

avec

$$
\alpha_{1}=4 \pi \int_{\mathbb{R}^{3}}\left(V_{+}+V_{-}\right)(x) \mathrm{d} x .
$$

Actuellement, l'existence d'un développement asymptotique complet n'est pas établie. Une différence importante avec l'opérateur de Schrödinger est que la norme (dans un espace à poids) de la résolvante au bord (définie par le principe d'absorption limite) ne temps pas vers zéro avec $1 / \sqrt{\lambda}$, elle est seulement bornée (cf. [12]).

Par contre, si $V=0$, on montre que l'étude de la phase de diffusion pour l'opérateur de Dirac avec champ magnétique se ramène facilement à celle des opérateurs du type Schrödinger réalisée par D. Robert ([15], [16]). Cela résulte des propriétés de supersymétrie que possède l'opérateur de Dirac avec champ magnétique. Dans ce cas, on a alors un développement asymptotique complet.

THÉORÈme 2.3. - On suppose que $V$ est nul et que $A$ vérifie $\left(H_{\delta}\right)$, avec $\delta>3$. On a alors l'asymptotique complète suivante :

$$
\frac{\mathrm{d} s}{\mathrm{~d} \lambda}(\lambda) \asymp(2 \pi)^{-3} \lambda^{-2} \sum_{j \geq 0} \beta_{j} \lambda^{-2 j}, \quad \lambda \rightarrow \pm \infty,
$$

avec

$$
\beta_{0}=\frac{8 \pi}{3} \int_{\mathbb{R}^{3}} B^{2}(x) \mathrm{d} x
$$

où $B=\operatorname{rot}(A)$ est le champ magnétique. De plus, cette asymptotique est dérivable à tout ordre par rapport $\grave{a} \lambda$.

\section{Asymptotique faible}

Pour la démonstration du théorème 2.1, nous renvoyons aux méthodes standards de calcul fonctionnel et de calcul de trace déjà employées pour des opérateurs de type Schrödinger ([10], [13], [6], [15], etc.). 
Notons cependant que la principale différence est que $H$ est de symbole principal matriciel non semi-borné. Nous précisons donc comment établir que $\phi(h H)$ est $h$-admissible et comment calculer son symbole.

Pour démontrer que $\phi(h H)$ est $h$-admissible, une première méthode consiste à utiliser les extensions presque analytiques comme le suggèrent B. Helffer et J. Sjöstrand [11]. Celle-ci permet de relier $\phi(h H)$ directement à la résolvante $(h H-z)^{-1}$.

Une autre solution est de séparer les énergies positives et négatives, grâce à la relation suivante :

$$
\phi(\lambda)=\frac{1}{2}\left(1+\frac{\lambda}{|\lambda|}\right) \phi(|\lambda|)+\frac{1}{2}\left(1-\frac{\lambda}{|\lambda|}\right) \phi(-|\lambda|), \quad \text { si } \lambda \neq 0 .
$$

Ainsi, l'étude de $\phi(h H)$ se ramène au calcul fonctionnel de $h^{2} H^{2}$ et à la composition par $h H$. Le symbole principal de $H^{2}$ étant de type scalaire $a_{0}^{2}(\xi)=(\alpha \cdot \xi)^{2}=|\xi|^{2} I_{4}$, on utilise les études déjà réalisées pour des perturbations du laplacien ([6], [13], [14]).

On établit ainsi que $\phi(h H)$ est $h$-admissible, c'est-à-dire que son symbole $a_{\phi}$ admet un développement en puissances de $h$ :

$$
a_{\phi} \sim \sum_{\ell \geq 0} h^{\ell} a_{\phi, \ell}
$$

Pour l'expression des coefficients, nous reprenons la méthode de Seeley [18], utilisée dans des contextes voisins dans [13], [10], [6], [15]. Cependant, la non-commutativité des symboles matriciels complique un peu la détermination des $a_{\phi, l}$. Avant d'effectuer quelques calculs, donnons un lemme.

LEMME 3.1. - Soit a un symbole matriciel (d'ordre 4) et $a_{0}(\xi)=\alpha \cdot \xi$, le symbole principal de $H$. Pour toute fonction définie sur $\mathbb{R}$, on a

$$
\begin{gathered}
f\left(a_{0}\right) a=a_{D} f\left(a_{0}\right)+a_{M} f\left(-a_{0}\right), \\
\text { où } a_{D}=P^{+} a P^{+}+P^{-} a P^{-} \text {et } a_{M}=P^{+} a P^{-}+P^{-} a P^{+}, \\
P^{ \pm} \stackrel{\text { def }}{=} \frac{1}{2}\left(1 \pm \frac{\alpha \cdot \xi}{|\xi|}\right)
\end{gathered}
$$

étant la projection orthogonale sur le sous-espace propre associé à la valeur propre $\pm|\xi|$ de $\alpha \cdot \xi$. 
Asymptotique de la phase de diffusion à haute énergie pour l'opérateur de Dirac

Preuve. - Les sous-espaces propres associés aux valeurs propres $\pm|\xi|$ de $\alpha \cdot \xi$ sont supplémentaires, donc

$$
f\left(a_{0}\right) a=f\left(a_{0}\right) P^{+} a+f\left(a_{0}\right) P^{-} a .
$$

Or, par définition de $P^{ \pm}$, on a $f\left(a_{0}\right) P^{ \pm}=f( \pm|\xi|) P^{ \pm}$. Comme $f( \pm|\xi|)$ est un scalaire, il commute avec toute matrice, et donc

$$
f\left(a_{0}\right) a=P^{+} a f(|\xi|)+P^{-} a f(-|\xi|) .
$$

On obtient alors le résultat en utilisant que

$$
f( \pm|\xi|)=P^{+} f\left( \pm a_{0}\right)+P^{-} f\left(\mp a_{0}\right) \text {. }
$$

Le calcul des coefficients $a_{\phi, \ell}$ repose sur la construction d'une paramétrixe pour $(h H-z)^{-1}$, c'est-à-dire d'une famille de symboles $\left(q_{\ell, z}\right)_{\ell \geq 0}$. De façon classique, celle-ci est construite par récurrence en posant :

$$
\begin{aligned}
q_{0, z}(x, \xi) & =\left(a_{0}(x, \xi)-z\right)^{-1} \\
q_{\ell, z}(x, \xi) & =-q_{0, z} \times \\
& \times \sum_{k=0}^{\ell-1} \sum_{j+k+|\alpha+\beta|=\ell} \Gamma(\alpha, \beta)\left(\partial_{\xi}^{\beta} \mathrm{D}_{x}^{\alpha} a_{j}\right)\left(\partial_{\xi}^{\alpha} \mathrm{D}_{x}^{\beta} q_{k, z}\right), \text { pour } \ell \geq 1
\end{aligned}
$$

où

$$
\Gamma(\alpha, \beta)=\frac{(-1)^{|\beta|}}{2^{|\alpha+\beta|} \alpha ! \beta !} .
$$

Le coefficient de $\phi^{(j)}\left(a_{0}\right)$ dans $a_{\phi, \ell}$ est donné par le coefficient de $\left(a_{0}-z\right)^{-j-1}$ dans $q_{\ell, z}$ (à un facteur $(-1)^{j}(j !)^{-1}$ près).

Ainsi, on obtient que $a_{\phi, 0}=\phi\left(a_{0}\right)$ et $a_{\phi, 1}$ est donné par (2) grâce au lemme suivant.

LEMME 3.2

$$
q_{1, z}=-a_{1, D}\left(a_{0}-z\right)^{-2}+\frac{1}{2} a_{1, M} a_{0}^{-1}\left(\left(a_{0}-z\right)^{-1}-\left(-a_{0}-z\right)^{-1}\right) .
$$


Preuve. - Par définition, on a

$$
q_{1, z}=-q_{0, z} a_{1} q_{0, z}-\frac{1}{2 i}\left\{a_{0}, q_{0, z}\right\}
$$

où $\{\cdot, \cdot\}$ désigne le crochet de Poisson. Comme ni $a_{0}$, ni $q_{0, z}$ ne dépend de $x$, le crochet de Poisson est nul et on a

$$
q_{1, z}=-\left(a_{0}-z\right)^{-1} a_{1}\left(a_{0}-z\right)^{-1} .
$$

Appliquons maintenant le lemme 3.1 à $a=a_{1}$, on obtient

$$
q_{1, z}=-a_{1, D}\left(a_{0}-z\right)^{-2}-a_{1, M}\left(-a_{0}-z\right)^{-1}\left(a_{0}-z\right)^{-1} .
$$

Le lemme résulte alors de l'équation de la résolvante.

\section{Formule de Weyl}

Pour démontrer le théorème 2.2, nous nous inspirons de [15] où $\mathrm{D}$. Robert montre une formule de type Weyl pour des perturbations du laplacien. Comme pour le sens faible, le fait que l'opérateur soit matriciel et non semi-borné nécessite des adaptations.

Avant de démontrer la formule de Weyl, donnons une propriété de symétrie qui permet de limiter notre étude aux énergies positives.

Proposition 4.1.- On suppose $\left(H_{\delta}\right)$ avec $\delta>3$. Soit $s_{-}$la phase de diffusion associée aux potentiels $(-A,-\bar{V})$ où

$$
\bar{V}=\left(\begin{array}{cc}
V_{-} 1_{2} & 0 \\
0 & V_{+} 1_{2}
\end{array}\right) \text {. }
$$

On a la propriété de symétrie :

$$
s_{-}(\lambda)=-s(-\lambda)
$$

Preuve. - Cette propriété résulte de la cyclicité de la trace et de l'existence d'un opérateur $C$ (opérateur de configuration de charge), unitaire $\operatorname{sur} L^{2}\left(\mathbb{R}^{3}, \mathbb{R}^{4}\right)$, tel que :

$$
C H C^{-1}=H_{-},
$$

où $H_{-}$est l'opérateur de Dirac associé au potentiel $(-A,-\bar{V})$ (cf. [19, $\S 1.4 .6])$. 
Asymptotique de la phase de diffusion à haute énergie pour l'opérateur de Dirac

Formellement, $s(\lambda)$ s'exprime en fonction de la différence des projecteurs spectraux de $H$ et $H_{0}$. Comme dans [15], nous commençons par l'exprimer uniquement en fonction du projecteur spectral de $H$. Introduisons

$$
Q:=H^{2}-H_{0}^{2}, \quad \mathcal{A}:=\frac{1}{2}(x \cdot \mathrm{D}+\mathrm{D} \cdot x) .
$$

L'opérateur différentiel $Q$ est d'ordre 1 à coefficients matriciels décroissants comme $A$ et $V\left(\mathrm{cf} .\left(\mathrm{H}_{\delta}\right)\right)$. Ici, le générateur des dilatations $\mathcal{A}$ est considéré comme opérateur agissant sur $L^{2}\left(\mathbb{R}^{3}, \mathbb{C}^{4}\right)$.

Proposition 4.2.- On suppose $\left(H_{\delta}\right)$ avec $\delta>3$. Soit

$$
f \in C_{0}^{\infty}(]-\infty,-1[\cup] 1,+\infty[),
$$

on a

$$
\int_{\mathbb{R}} s(\lambda) f^{\prime}(\lambda) \mathrm{d} \lambda=\operatorname{Tr}\left(\left(Q-\frac{i}{2}[Q, \mathcal{A}]\right)\left(H^{2}-1\right)^{-1} f(H)\right) .
$$

Preuve. - Ce résultat repose sur la relation $i[-\Delta, \mathcal{A}]=-2 \Delta$ et sur la cyclicité de la trace. En effet, on a

$$
2\left(H_{0}^{2}-I\right)=i\left[H_{0}^{2}, \mathcal{A}\right] \text { et } 2\left(H^{2}-I\right)=i\left[H^{2}, \mathcal{A}\right]+(2 Q-i[Q, \mathcal{A}]),
$$

et la trace étant cyclique [15, appendice $\mathrm{A}]$, on obtient

$$
\operatorname{Tr}\left(f(H)-f\left(H_{0}\right)\right)=\operatorname{Tr}\left(\left(Q-\frac{i}{2}[Q, \mathcal{A}]\right)\left(H^{2}-1\right)^{-1} f(H)\right) .
$$

La formule de Krein donne alors le résultat.

Il résulte de cette proposition une expression de $s(\lambda)$ en fonction de $E_{H}\left(\lambda_{0}, \lambda\right)\left(\lambda>\lambda_{0}>1\right)$, le projecteur spectral associé à $H$, sur l'intervalle $\left[\lambda_{0}, \lambda\right]$.

Corollatre 4.3. - Soit $\chi \in C^{\infty}(\mathbb{R})$ une fonction de troncature valant 1 sur $[2,+\infty[$ et nulle sur $]-\infty, 3 / 2]$. Alors, pour tout $\lambda>\lambda_{0}>2$, nous avons

$$
s(\lambda)-s\left(\lambda_{0}\right)=\operatorname{Tr}\left(\left(Q-\frac{i}{2}[Q, \mathcal{A}]\right) \frac{\chi(H)}{\left(H^{2}-1\right)} E_{H}\left(\lambda_{0}, \lambda\right)\right) .
$$


Pour étudier la phase de diffusion en $\lambda=+\infty$, nous introduisons un paramètre $h>0$. Pour $\mu$ dans un compact de ] $0,+\infty$ [, on pose

$$
s_{h}(\mu) \stackrel{\text { def }}{=} s\left(\frac{\mu}{h}\right)-s\left(\lambda_{0}\right)
$$

et nous nous intéressons au comportement de $s_{h}(\mu)$ en $h=0^{+}$, pour $\mu$ fixé.

On décompose $s_{h}$ en une partie décrivant le bas du spectre continu et une autre localisée autour de $\mu / h$ :

Soient $\chi_{1}$ et $\chi_{2}$ deux fonctions positives de $C_{0}^{\infty}(\mathbb{R})$, telles que $\chi_{1}+\chi_{2}=1$ sur $\left.\left.\left.\left.[0,3], \operatorname{supp} \chi_{1} \subset\right]-\infty, 1\right], \operatorname{supp} \chi_{2} \subset\right] 0,4\right]$. Alors, il existe $h_{0}>0$ tel que pour tout $\left.h \in] 0, h_{0}\right]$ et tout $\mu \in[1,3]$, on ait une décomposition de $s_{h}(\mu)$ :

$$
s_{h}(\mu)=s_{1, h}(\mu)+s_{2, h}(\mu)
$$

avec

$$
\begin{aligned}
& s_{1, h}(\mu) \stackrel{\text { def }}{=} \operatorname{Tr}\left(\left(Q-\frac{i}{2}[Q, \mathcal{A}]\right) \chi(H)\left(H^{2}-1\right)^{-1} \chi_{1}(h H)\right) \\
& s_{2, h}(\mu) \stackrel{\text { def }}{=} \operatorname{Tr}\left(\left(Q-\frac{i}{2}[Q, \mathcal{A}]\right) \chi_{2}(h H)\left(H^{2}-1\right)^{-1} E_{h}(\mu)\right)
\end{aligned}
$$

où $E_{h}$ est la projection spectrale associée à $h H$.

Comme $s_{1, h}$ ne dépend pas de la projection spectrale, le calcul fonctionnel pour des fonctions régulières nous donne l'existence d'un scalaire $\alpha_{1,0}$ dépendant des potentiels $A$ et $V$, tel que

$$
s_{1, h}=h^{-2} \alpha_{1,0}+\mathrm{O}\left(h^{-1}\right) \text {. }
$$

Pour l'étude de $s_{2, h}$, nous nous inspirons d'études antérieures du spectre ponctuels [9], et du spectre continu pour l'opérateur de Schrödinger [15]. L'idée est d'appliquer un théorème taubérien de [13, théorème V. 13] et de procéder comme dans [10] et [17]. Pour cela, on commence par ramener l'étude de $s_{2, h}$ à celle de fonctions croissantes du type

$$
s_{W, h}(\mu):=h \operatorname{Tr}\left(W(h) \chi_{2}(h H) E_{h}(\mu)\right),
$$

avec $W(h)$ opérateur positif, $h$-admissible, borné, de poids $\langle x\rangle^{-\delta}$. Il suffit de décomposer $s_{2, h}$ sous la forme:

$$
\begin{gathered}
s_{2, h}(\mu)=s_{W^{+, h}}(\mu)-s_{W^{-, h}}(\mu) \\
-216-
\end{gathered}
$$


Asymptotique de la phase de diffusion à haute énergie pour l'opérateur de Dirac

avec

$$
W^{+}(h)=2 M\langle x\rangle^{-\delta} \quad, \quad W^{-}(h)=\langle x\rangle^{-\delta / 2}(2 M-A(h))\langle x\rangle^{-\delta / 2}
$$

où

$$
M=\sup _{0<h \leq h_{0}}\|A(h)\| \text { et } A(h)=\langle x\rangle^{\delta / 2} R(h)\langle x\rangle^{\delta / 2} \text {, }
$$

$R(h)$ étant un opérateur, $h$-admissible, borné, de poids $\langle x\rangle^{-\delta}$ tel que

$$
R(h) \chi_{2}(h H)=h\left(Q-\frac{i}{2}[Q, \mathcal{A}]\right)\left(h^{2} H^{2}-h^{2}\right)^{-1} \chi_{2}(h H) \text {. }
$$

Le support de $\chi_{2}$ étant inclus dans $\left.] 0,4\right], s_{W, h}$ est nulle pour $\mu \leq 0$ et est constante pour $\mu \geq 4$. D'autre part, il résulte du calcul fonctionnel (théorème 2.1) et des théorèmes de traces d'opérateurs $h$-admissib ss [13], que l'on a

$$
s_{W, h}(\mu)=\mathrm{O}\left(h^{-2}\right), \quad h \rightarrow 0,
$$

uniformément par rapport à $\mu \in \mathbb{R}$.

Pour appliquer le théorème taubérien, il reste alors à étudier le comportement asymptotique, quand $h$ tend vers 0 de

$$
\frac{\mathrm{d}}{\mathrm{d} \mu}\left(s_{W, h} * \theta_{h}\right)(\mu),
$$

où $\theta_{h}$ est une fonction régularisante définie de la façon suivante : soit $\zeta \in C_{0}^{\infty}(]-T, T[),(T>0$ fixé $)$, tel que $\zeta(0)=1$. La fonction $\theta_{h}$ est définie par

$$
\theta_{h}(\mu):=\frac{1}{2 \pi h} \widehat{\zeta}\left(\frac{\mu}{h}\right), \quad \mu \in \mathbb{R}
$$

Avant son étude rigoureuse, nous donnons une interprétation formelle de $(\mathrm{d} / \mathrm{d} \mu)\left(s_{W, h} * \theta_{h}\right)(\mu)$. De l'expression de $s_{W, h}$, on déduit formellement une expression de $s_{W, h}^{\prime}$,

$$
\frac{\mathrm{d}}{\mathrm{d} \mu} s_{W, h}=h \operatorname{Tr}\left(W(h) \chi_{2}(h H) \frac{\mathrm{d} E_{h}}{\mathrm{~d} \mu}\right) .
$$

Or, la formule de Stone donne, formellement,

$$
\begin{gathered}
\frac{\mathrm{d} E_{h}}{\mathrm{~d} \mu}(\lambda)=\frac{1}{2 \pi h} \int_{\mathbb{R}} e^{i t(h H-\lambda) / h} \mathrm{~d} t \\
-217-
\end{gathered}
$$




\section{Vincent Bruneau}

donc on peut espérer approcher $s_{W, h}^{\prime}$ par

$$
\frac{h}{2 \pi h} \operatorname{Tr}\left(W(h) \chi_{2}(h H) \int_{\mathbb{R}} \zeta(t) e^{i t(h H-\lambda) / h} \mathrm{~d} t\right)
$$

avec $\zeta \in C_{0}^{\infty}(\mathbb{R})$, égale à 1 au voisinage de 0 . Mais cette quantité correspond à la convolution de $s_{W, h}^{\prime}$ avec

$$
\theta_{h}=\frac{1}{2 \pi h} \widehat{\zeta}\left(\frac{\cdot}{h}\right) .
$$

C'est ce qui justifie, formellement, l'introduction de $\theta_{h}$.

Pour étudier la convolution, nous utilisons l'approximation du propagateur donnée au paragraphe suivant, et en procédant de façon analogue à [10] et [17], on établit la proposition suivante.

Proposition 4.4. - Sous l'hypothèse $\left(H_{\delta}\right)$ avec $\delta>3$,

$$
\frac{\mathrm{d}}{\mathrm{d} \mu}\left(s_{W, h} * \theta_{h}\right)(\mu)
$$

admet un développement asymptotique complet :

$$
\frac{\mathrm{d}}{\mathrm{d} \mu}\left(s_{W, h} * \theta_{h}\right)(\mu) \asymp h^{-2} \sum_{j \geq 0} C_{j, W}(\zeta, \mu) h^{j}, \quad h \rightarrow 0 .
$$

De plus, les distributions $\zeta \mapsto C_{j, W}(\zeta, \mu)$ ne dépendent que du germe de $\zeta$ à l'origine et les fonctions $\mu \mapsto C_{j, W}(\zeta, \mu)$ appartiennent à $C_{0}^{\infty}(\mathbb{R})$.

Preuve. - Par définition de $s_{W, h}$ (cf. (6)), on a

$$
\frac{\mathrm{d}}{\mathrm{d} \mu}\left(s_{W, h} * \theta_{h}\right)(\mu)=\frac{h}{2 \pi h} \operatorname{Tr}\left(W(h) \chi_{2}(h H) \int_{\mathbb{R}} \zeta(t) e^{-i t \mu / h} e^{i t H} \mathrm{~d} t\right)
$$

En appliquant la proposition 5.1 donnant l'approximation du propagateur par un O.F.I., il vient, $\forall T>0, \forall \zeta \in C_{0}^{\infty}(]-T, T[)$ et $\forall N>0$, on a

$$
\left|\frac{\mathrm{d}}{\mathrm{d} \mu}\left(s_{W, h} * \theta_{h}\right)(\mu)-\tau_{W, h}^{N}(\mu)\right|=\mathrm{O}\left(h^{N-3}\right),
$$

où

$$
\tau_{W, h}^{N}(\mu) \stackrel{\text { def }}{=} \frac{h}{2 \pi h} \operatorname{Tr}\left(W(h) \chi_{2}(h H) \int_{\mathbb{R}} \zeta(t) e^{-i t \mu / h} \tilde{U}_{h}^{N}(t) \mathrm{d} t\right)
$$


Asymptotique de la phase de diffusion à haute énergie pour l'opérateur de Dirac

Il suffit donc de montrer l'existence du développement asymptotique pour $\tau_{W, h}^{N}(\mu)$. Posons :

$$
G_{W, h}^{N}(t) \stackrel{\text { def }}{=} \operatorname{Tr}\left(\chi_{2}(h H) W(h) \tilde{U}_{h}^{N}(t)\right) .
$$

Par définition de $\widetilde{U}_{h}^{N}(t)(11)$, on a

$$
G_{W, h}^{N}(t)=G_{W, h}^{N,+}(t)+G_{W, h}^{N,-}(t)
$$

où

$$
G_{W, h}^{N, \pm}(t) \stackrel{\text { def }}{=} \operatorname{Tr}\left(\chi_{2}(h H) W(h) \tilde{U}_{h}^{N, \pm}(t)\right)
$$

En utilisant les théorèmes généraux relatifs à la composée d'un opérateur pseudo-différentiel par un O.F.I. (cf. par exemple [13, théorème IV-24]), et au calcul de trace d'O.F.I. (cf. par exemple [8, chap. III.10]), les contructions réalisées à la section suivante donnent que $\tau_{W, h}^{N}=\tau_{W, h}^{N,+}+\tau_{W, h}^{N,-}$ avec

$$
\tau_{W, h}^{N, \pm}(\mu)=\frac{h}{(2 \pi h)^{4}} \sum_{j=0}^{N} h^{j} \int_{0}^{+\infty} \int_{\mathbb{R}} \zeta(t) e^{-i t(\mu \mp r) / h} r^{2} \theta_{j}^{ \pm}(t, r) \mathrm{d} t \mathrm{~d} r
$$

où les $\theta_{j}^{ \pm}$sont bornées, appartiennent à $C_{b}^{\infty}\left(\mathbb{R}_{t} \times \mathbb{R}_{r}^{+}\right)$et sont à support compact par rapport à $r$. Nous étudions le comportement asymptotique, quand $h \searrow 0$, seulement dans le cas $\mu>0$, le cas $\mu<0$ étant tout à fait similaire (il suffit de changer les signes).

La phase $\varphi^{-}(t, r)=t(\mu+r)$ ne possède aucun point stationnaire, donc, d'après le théorème de la phase non stationaire, on a

$$
\tau_{W, h}^{N,-}(\mu)=\mathrm{O}\left(h^{\infty}\right) \text { lorsque } h \searrow 0 \text { pour } \mu>0 .
$$

Par contre, la phase $\varphi^{+}(t, r)=t(\mu-r)$ admet un point stationnaire $(0, \mu)$, le théorème de la phase non stationnaire donne, pour $\mu>0$,

$$
\tau_{W, h}^{N,+}(\mu) \asymp \frac{h^{-2}}{(2 \pi)^{3}} \mu^{2}\left(\theta_{0}^{+}(0, \mu)+\sum_{j \geq 1} h^{j} C_{j}^{+}(\zeta, \mu)\right), \quad h \searrow 0
$$

où $\zeta \mapsto C_{j}^{+}(\zeta, \mu)$ est une distribution ne dépendant que du germe de $\zeta$ à l'origine et les fonctions $\mu \mapsto C_{j, W}^{+}(\zeta, \mu)$ appartiennent à $C_{0}^{\infty}(\mathbb{R})$. D'où la proposition, grâce à (7). 
De cette proposition, nous déduisons

$$
\frac{\mathrm{d}}{\mathrm{d} \mu}\left(s_{W, h} * \theta_{h}\right)(\mu)=\mathrm{O}\left(h^{-2}\right), \quad\left(s_{W, h} * \theta_{h}\right)(\mu)=h^{-2} \widetilde{C}_{0, W}(\mu)+\mathrm{O}\left(h^{-1}\right),
$$

quand $h$ tend vers 0 , localement uniformément par rapport à $\mu$, où la fonction $\mu \mapsto \widetilde{C}_{0, W}(\mu)$ est de classe $C^{\infty}$.

Le théorème taubérien [13, théorème V.13] donne alors :

$$
s_{W, h}(\mu)=h^{-2} \widetilde{C}_{0, W}(\mu)+\mathrm{O}\left(h^{-1}\right) .
$$

Appliqué à $W=W^{ \pm}$, ce résultat permet d'obtenir l'existence d'une fonction $\alpha_{2,0} \in C^{\infty}(\mathbb{R})$ telle que

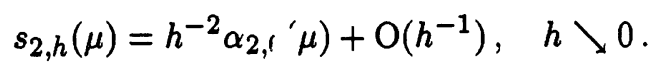

Grâce à (4) et (5), on déduit alc s le théorème 2.2. Le coefficient $\alpha_{1}$ est donné par le développement au séis faible (théorème 2.1).

\section{Approximation du propagateur à temps petit}

Dans la démonstration de la proposition 4.4, nous avons eu besoin de la proposition suivante, dont nous donnons maintenant la démonstration.

Proposition 5.1. - Soit $U(t):=e^{i t H}$ le propagateur associé à l'opérateur de Dirac $H$. Il existe un famille d'opérateurs $\left\{\widetilde{U}_{h}^{N}(t)\right\}_{t, N, h}, t \in \mathbb{R}$, $\left.N \in \mathbb{N}, h \in] 0, h_{0}\right]$ de sorte que pour tout $\chi \in C_{0}^{\infty}(\mathbb{R} \backslash 0)$, pour tout $N \in \mathbb{N}$ et $T>0$, on ait

$$
\left\|U(t) \chi(h H)-\tilde{U}_{h}^{N}(t) \chi(h H)\right\|=\mathrm{O}\left(h^{N-3}|t|\right), \quad \text { pour } t \in[-T, T] .
$$

De plus, $\widetilde{U}_{h}^{N}(t)$ est de la forme

$$
\widetilde{U}_{h}^{N}(t)=\tilde{U}_{h}^{N,+}(t)+\widetilde{U}_{h}^{N,-}(t),
$$

où $\widetilde{U}_{h}^{N, \pm}(t)$ est un O.F.I. de phase $\pm t|\xi| \widetilde{\chi}(|\xi|)+x \cdot \xi$, avec $\tilde{\chi} \in C^{\infty}\left(\mathbb{R}^{+} \backslash 0\right)$ telle que $\tilde{\chi} \chi=\chi$ et d'amplitude une matrice $4 \times 4$ polynômiale, d'ordre $N$, par rapport à $h$. 
Asymptotique de la phase de diffusion à haute énergie pour l'opérateur de Dirac

On cherche à approcher le propagateur, $U(t)$, par un O.F.I, $U_{h}^{N}(t)$, de noyau intégral de la forme

$$
U_{h}^{N}(t ; x, y)=(2 \pi h)^{-3} \int_{\mathbb{R}^{3}} \exp \left(-\frac{i}{h}(S(t, x, \xi)-y \cdot \xi)\right) \sum_{j=0}^{N} h^{j} b_{j}(t, x, \xi) \mathrm{d} \xi
$$

avec des amplitudes $b_{j}$ matricielles. Pour cela, on utilise que $U(t)$ est l'opérateur qui à une fonction $\phi$ associe la solution $\psi$ de l'équation de Dirac :

$$
\begin{aligned}
& i \partial_{t} \psi(t, x)+H \psi(t, x)=0 \\
& \psi(0, x)=\phi(x)
\end{aligned}
$$

En substituant $U_{h}^{N}(t ; x, y)$ ( $y$ fixé) dans (8) et en ordonnant suivant les puissances croissantes de $h$, l'annulation des coefficients donne

$$
\begin{aligned}
& 0=\left(\partial_{t} S I_{4}-\alpha \cdot \nabla_{x} S\right) b_{0} \\
& i \partial_{t} b_{j}+H_{x} b_{j}=\left(\partial_{t} S I_{4}-\alpha \cdot \nabla_{x} S\right) b_{j+1} \quad \text { pour } j \geq 0 .
\end{aligned}
$$

$H_{x}$ désigne l'opérateur de Dirac, l'indice $x$ rappelle que c'est un opérateur différentiel par rapport à la variable $x$. La condition (9) sera obtenue en demandant

$$
S(0, x, \xi)=x \cdot \xi, \quad b_{0}(0, x, \xi)=I_{4} \quad \text { et } \quad b_{j}(0, x, \xi)=0 \quad \text { pour } j \geq 1
$$

Pour résoudre ces systèmes, nous nous inspirons des méthodes utilisées pour les systèmes strictement hyperboliques (cf. par exemple [7, chap. 2]).

On déduit de (10) que sur le support de la fonction matricielle $b_{0}$, on a l'équation eikonale :

$$
\operatorname{det}\left(\partial_{t} S I_{4}-\alpha \cdot \nabla_{x} S\right)=0
$$

Cette équation admet deux solutions vérifiant $S(0, x, \xi)=x \cdot \xi$, correspondant aux deux valeurs propres $( \pm|\xi|)$ de $\alpha \cdot \xi$ :

$$
S^{ \pm} \stackrel{\text { def }}{=} \pm t|\xi|+x \cdot \xi .
$$




\section{Vincent Bruneau}

Comme K. Yajima dans [20] (cas semi-classique), on cherche donc $U_{h}^{N}(t)$ de la forme

$$
U_{h}^{N}(t)=U_{h}^{+, N}(t)+U_{h}^{-, N}(t)
$$

où $U_{h}^{ \pm, N}(t)$ sont des O.F.I. de noyau intégral de la forme

$$
\begin{aligned}
& G_{h}^{ \pm, N}(t ; x, y)= \\
& \quad=(2 \pi h)^{-3} \int_{\mathbb{R}^{3}} \exp \left(-\frac{i}{h}\left(S^{ \pm}(t, x, \xi)-y \cdot \xi\right)\right) \sum_{j=0}^{N} h^{j} b_{j}^{ \pm}(t, x, \xi) \mathrm{d} \xi
\end{aligned}
$$

avec les $b_{j}^{\ddagger}$ vérifiant les équations de transport

$$
\begin{aligned}
& \left(\partial_{t} S^{ \pm} I_{4}-\alpha \cdot \nabla_{x} S^{ \pm}\right) b_{0}^{ \pm}=0 \\
& \left(i \partial_{t}+H_{x}\right) b_{j}^{ \pm}=\left(\partial_{t} S^{ \pm} I_{4}-\alpha \cdot \nabla_{x} S^{ \pm}\right) b_{j+1}^{ \pm}, \quad j \geq 0 \\
& b_{0}^{+}(0, x, \xi)+b_{0}^{-}(0, x, \xi)=I_{4} \\
& b_{j}^{+}(0, x, \xi)+b_{j}^{-}(0, x, \xi)=0, \quad j \geq 1 .
\end{aligned}
$$

Or, par définition de $S^{ \pm}$, on a

$$
\left(\partial_{t} S^{ \pm} I_{4}-\alpha \cdot \nabla_{x} S^{ \pm}\right)(t, x, \xi)= \pm 2|\xi| P^{\mp}(\xi)
$$

où

$$
P^{ \pm}(\xi) \stackrel{\text { def }}{=} \frac{1}{2}\left(1 \pm \frac{\alpha \cdot \xi}{|\xi|}\right)
$$

sont les projections orthogonales sur les sous-espaces propres $\mathcal{E}^{ \pm}$associés aux valeurs propres doubles $( \pm|\xi|)$ de la matrice $\alpha \cdot \xi$.

Ainsi, après projection sur les espaces complémentaires $\mathcal{E}^{+}$et $\mathcal{E}^{-}$, le système (12)-(15) est équivalent à

$$
\begin{aligned}
& |\xi| P^{-\varepsilon} b_{0}^{\varepsilon}=0 \\
& P^{\varepsilon}\left(i \partial_{t}+H_{x}\right) b_{j}^{\varepsilon}=0, \quad j \geq 0 \\
& P^{-\varepsilon}\left(i \partial_{t}+H_{x}\right) b_{j}^{\varepsilon}=2 \varepsilon|\xi| P^{-\varepsilon} b_{j+1}^{\varepsilon}, \quad j \geq 0 \\
& b_{0}^{+}(0, x, \xi)+b_{0}^{-}(0, x, \xi)=I_{4} \\
& b_{j}^{+}(0, x, \xi)+b_{j}^{-}(0, x, \xi)=0, \quad j \geq 1
\end{aligned}
$$

où, pour allèger l'écriture, on a posé $\varepsilon= \pm$ et $-\varepsilon=\mp$. 
Asymptotique de la phase de diffusion à haute énergie pour l'opérateur de Dirac

Avant de résoudre le système (16)-(20), notons la relation suivante :

$$
P^{\varepsilon} H_{x}=\left(\alpha \cdot \mathrm{d}_{A}+\varepsilon \frac{\xi}{|\xi|} \cdot \mathrm{d}_{A}+\beta \widetilde{v}\right) P^{-\varepsilon}+\left(v+\varepsilon \frac{\xi}{|\xi|} \cdot \mathrm{d}_{A}\right) P^{\varepsilon}
$$

où $\mathrm{d}_{A}=\mathrm{D}_{x}-A, v=\left(V_{+}+V_{-}\right) / 2$ et $\tilde{v}=1+\left(V_{+}-V_{-}\right) / 2$. Cette relation résulte des propriétés des matrices de Dirac

$$
\alpha_{i} \alpha_{j}+\alpha_{j} \alpha_{i}=2 \delta_{i j} I_{4}, \quad \text { pour } i, j=1,2,3,4, \alpha_{4}=\beta
$$

en remarquant que $H_{x}$ s'écrit

$$
H_{x}=\alpha \cdot \mathrm{d}_{A}+\beta \widetilde{v}+v
$$

Nous résolvons maintenant le système (16)-(20) de proche en proche. D'après (16), $b_{0}^{\varepsilon}$ est dans $\mathcal{E}^{\varepsilon}$, l'orthogonal de $\mathcal{E}^{-\varepsilon}$. Ainsi, il existe une matrice $d_{0}^{\varepsilon}$ telle que

$$
b_{0}^{\varepsilon}(t, x, \xi)=P^{\varepsilon}(\xi) d_{0}^{\varepsilon}(t, x, \xi)
$$

Grâce à la relation (21), l'équation (17) s'écrit donc, pour $j=0$,

$$
P^{\varepsilon}\left(i \partial_{t}+v+\varepsilon \frac{\xi}{|\xi|} \cdot \mathrm{d}_{A}\right) d_{0}^{\varepsilon}=0
$$

et (19) sera satisfait si $d_{0}^{\varepsilon}(0, x, \xi)=I_{4}$. Il suffit donc de chercher $d_{0}^{\varepsilon}$ sous forme scalaire solution de

$$
\begin{aligned}
& \left(i \partial_{t}+v(x)-\varepsilon \frac{\xi}{|\xi|} \cdot A(x)+\varepsilon \frac{\xi}{|\xi|} \cdot \mathrm{D}_{x}\right) d_{0}^{\varepsilon}(t, x, \xi)=0 \\
& d_{0}^{\varepsilon}(0, x, \xi)=1
\end{aligned}
$$

La résolution de ce problème de Cauchy donne

$$
d_{0}^{\varepsilon}(t, x, \xi)=\exp \left(i \int_{0}^{t}\left(v-\varepsilon \frac{\xi}{|\xi|} \cdot A\right)\left(x+\varepsilon \frac{\xi}{|\xi|} \tau\right) \mathrm{d} \tau\right)
$$

Ainsi, on obtient le lemme suivant. 


\section{Vincent Bruneau}

LEMME 5.2

- Le système (16)-(20) admet une solution $b_{0}^{\varepsilon}$ donnée par

$$
b_{0}^{\varepsilon}(t, x, \xi)=P^{\varepsilon}(\xi) \exp \left(i \int_{0}^{t}\left(v-\varepsilon \frac{\xi}{|\xi|} \cdot A\right)\left(x+\varepsilon \frac{\xi}{|\xi|} \tau\right) \mathrm{d} \tau\right) .
$$

- $b_{0}^{\varepsilon} \in C^{\infty}\left(\mathbb{R}_{t}, \mathbb{R}_{x}^{3}, \mathbb{R}_{\xi}^{3 *}\right)$ et $\forall(k, \alpha, \beta) \in \mathbb{N} \times \mathbb{N}^{3} \times \mathbb{N}^{3}, \forall T>0, \exists C_{k, \alpha, \beta, T}$ tel que

$\left|\partial_{t}^{k} \partial_{x}^{\alpha} \partial_{\xi}^{\beta} b_{0}^{\varepsilon}(t, x, \xi)\right| \leq C_{k, \alpha, \beta, T}\langle\xi\rangle^{-|\beta|}, \quad \forall(t, x, \xi) \in[-T, T] \times \mathbb{R}_{x}^{3} \times \mathbb{R}_{\xi}^{3 *}$.

Par récurrence, on obtient les solutions $\left(b_{j}^{\varepsilon}\right)_{j \geq 0}$ de $(16)-(20)$. Elles sont de la forme

$$
b_{j}^{\varepsilon}(t, x, \xi)=P^{\varepsilon} \lambda_{j}^{\varepsilon} d_{0}^{\varepsilon}(t, x, \xi)+P^{-\varepsilon} Z_{j}^{\varepsilon}(t, x, \xi)
$$

où $\lambda_{0}^{\varepsilon}=I_{4}, Z_{0}^{\varepsilon}=0$, et pour $j \geq 1$,

$$
\begin{aligned}
& Z_{j}^{\varepsilon}= \frac{\varepsilon}{2|\xi|} P^{-\varepsilon}\left(\left(\alpha \cdot \mathrm{d}_{A}+\varepsilon \frac{\xi}{|\xi|} \cdot \mathrm{d}_{A}+\beta \tilde{v}\right) \lambda_{j-1}^{\varepsilon} d_{0}^{\varepsilon}+\right. \\
&\left.+\left(i \partial_{t}+v+\varepsilon \frac{\xi}{|\xi|} \cdot \mathrm{d}_{A}\right) Z_{j-1}^{\varepsilon}\right) \\
& \lambda_{j}^{\varepsilon}=i \int_{0}^{t} I_{j}^{\varepsilon}\left(\tau, x+\varepsilon \frac{\xi}{|\xi|}(t-\tau), \xi\right) \mathrm{d} \tau-Z_{j}^{-\varepsilon}\left(0, x+\varepsilon \frac{\xi}{|\xi|} t, \xi\right),
\end{aligned}
$$

$I_{j}^{\varepsilon}$ étant défini par

$$
I_{j}^{\varepsilon} d_{0}^{\varepsilon}(t, x, \xi) \stackrel{\text { def }}{=}\left(\alpha \cdot \mathrm{d}_{A}-\varepsilon \frac{\xi}{|\xi|} \cdot \mathrm{d}_{A}+\beta \tilde{v}\right) Z_{j}^{\varepsilon}(t, x, \xi) .
$$

Ces solutions vérifient la proposition suivante.

Proposition 5.3.- Pour tout $j \geq 0, b_{j}^{\varepsilon} \in C^{\infty}\left(\mathbb{R}_{t}, \mathbb{R}_{x}^{3}, \mathbb{R}_{\xi}^{3 *}\right)$ et $\forall(k, \alpha, \beta) \in \mathbb{N} \times \mathbb{N}^{3} \times \mathbb{N}^{3}, \forall T>0, \exists C_{k, \alpha, \beta, T}$ tel que $\left|\partial_{t}^{k} \partial_{x}^{\alpha} \partial_{\xi}^{\beta} b_{j}^{\varepsilon}(t, x, \xi)\right| \leq C_{k, \alpha, \beta, T}\langle\xi\rangle^{-j-|\beta|}, \quad \forall(t, x, \xi) \in[-T, T] \times \mathbb{R}_{x}^{3} \times \mathbb{R}_{\xi}^{3 *}$.

Les fonctions $S^{ \pm}$et $\left(b_{j}^{ \pm}\right)_{j \geq 0}$ n'étant pas définies en $\xi=0$, nous ne pouvons pas définir directement des O.F.I. de phase $S^{ \pm}$et d'amplitude $b_{j}^{ \pm}$. 
Asymptotique de la phase de diffusion à haute énergie pour l'opérateur de Dirac

C'est pourquoi, nous définissons les fonctions $\widetilde{S}^{ \pm}$et $\tilde{b}_{j}^{ \pm}$comme troncatures de $S^{ \pm}$et $b_{j}^{\ddagger}$. À partir de ces fonctions, nous pourrons définir un O.F.I. qui approche le propagateur pour des énergies en dehors d'un voisinage de 0 .

Soit $\tilde{\chi} \in C^{\infty}(\mathbb{R})$ à support dans $] 0,+\infty[$ valant 1 sur $[1 / 2,+\infty[$ et telle que $0 \leq \tilde{\chi} \leq 1$. Soit $\tilde{\chi}_{1} \in C^{\infty}(\mathbb{R})$ nulle au voisinage de 0 et telle que $\tilde{\chi}_{1} \tilde{\chi}=\tilde{\chi}, 0 \leq \tilde{\chi}_{1} \leq 1$. Alors, on pose

$$
\begin{gathered}
\widetilde{S}^{ \pm}(t, x, \xi)= \pm t|\xi| \tilde{\chi}_{1}(|\xi|)+x \cdot \xi \\
\tilde{b}_{j}^{ \pm}(t, x, \xi)=\tilde{\chi}(|\xi|) b_{j}^{ \pm}(t, x, \xi) .
\end{gathered}
$$

Comme $S^{ \pm}$et $\left(b_{j}^{ \pm}\right)_{j \geq 0}$, les fonctions $\tilde{S}^{ \pm}$et $\tilde{b}_{j}^{ \pm}$vérifient (16)-(20), sauf que (19) est remplacée par

$$
\tilde{b}_{0}^{+}(0, x, \xi)+\tilde{b}_{0}^{-}(0, x, \xi)=\tilde{\chi}(|\xi|)
$$

D'après la théorie des opérateurs intégraux de Fourier, nous pouvons alors définir un opérateur $\widetilde{U}_{h}^{N}(t)$ par

$$
\tilde{U}_{h}^{N}(t):=\tilde{U}_{h}^{+, N}(t)+\tilde{U}_{h}^{-, N}(t)
$$

où $\widetilde{U}_{h}^{ \pm, N}(t)$ est un O.F.I. de phase $\widetilde{S}^{ \pm}$et d'amplitude $\sum_{j=0}^{N} h^{j} \widetilde{b}_{j}^{ \pm}(t, x, \xi)$.

Les estimations de la proposition 5.3, nous permettent de prolonger cet opérateur par un opérateur borné sur $L^{2}\left(\mathbb{R}^{3}\right)^{4}$, l'action de $\left(i \partial_{t}+H_{x}\right)$ sur $\widetilde{U}_{h}^{N}(t)$ définit aussi un O.F.I., et, par construction, nous avons la proposition suivante.

Proposition 5.4. - Soit $R_{h}^{N}(t) \stackrel{\text { def }}{=}\left(i \partial_{t}+H\right) \widetilde{U}_{h}^{N}(t)$.

a) $R_{h}^{N}(t)=R_{h}^{+, N}(t)+R_{h}^{-, N}(t)$ ò̀ $R_{h}^{ \pm, N}(t)$ est un O.F.I. de phase $\widetilde{S}^{ \pm}$ et d'amplitude $\left(i \partial_{t} \tilde{b}_{N}^{ \pm}-H_{x} \widetilde{b}_{N}^{ \pm}\right)$.

b) $R_{h}^{N}(t)$ se prolonge par un opérateur borné sur $L^{2}\left(\mathbb{R}^{3}\right)^{4}$ et pour tout $N \in \mathbb{N}, T>0$, il existe $C_{N, T}>0$ tel que

$$
\left\|R_{h}^{N}(t)\right\| \leq C_{N, T} h^{N-3} \quad \text { pour } t \in[-T, T] .
$$

En utilisant, de plus, que $\widetilde{U}_{h}^{N}(0)=\tilde{\chi}\left(h \mathrm{D}_{x}\right)$ (cf. (22)) et la formule de Duhamel,

$$
\tilde{U}_{h}^{N}(t)-U(t) \tilde{\chi}\left(h \mathrm{D}_{x}\right)=-i \int_{0}^{t} U(t-s) R_{h}^{N}(s) \mathrm{d} s,
$$

de ce qui précède on déduit que $\widetilde{U}_{h}^{N}(t)$ approche $U(t) \widetilde{\chi}\left(h \mathrm{D}_{x}\right)$. Le calcul fonctionnel donne alors la proposition 5.1. 


\section{Supersymétrie}

L'opérateur de Dirac avec uniquement un potentiel magnétique entre dans le cadre abstrait des opérateurs de Dirac supersymétriques. Il s'agit d'opérateurs qui, sur un espace $\mathcal{H}=\mathcal{H}_{+} \oplus \mathcal{H}_{-}$, sont de la forme

$$
H=\left(\begin{array}{cc}
\tilde{m} \mathrm{Id} & \mathcal{D}^{*} \\
\mathcal{D} & -\tilde{m} \mathrm{Id}
\end{array}\right)
$$

$\tilde{m}$ étant un réel strictement positif et $\mathcal{D}^{*}$ l'adjoint de $\mathcal{D}$.

L'avantage de ces opérateurs de Dirac est que leurs énergies positives et négatives sont bien séparées. Ceci se traduit par l'existence d'un opérateur unitaire $U_{F W}$ tel que (cf. [19, théorème 5.13])

$$
U_{F W}\left(\begin{array}{cc}
\tilde{m} \mathrm{Id} & \mathcal{D}^{*} \\
\mathcal{D} & -\tilde{m} \mathrm{Id}
\end{array}\right) U_{F W}^{*}=\left(\begin{array}{cc}
\sqrt{\mathcal{D}^{*} \mathcal{D}+\tilde{m}^{2}} & 0 \\
0 & -\sqrt{\mathcal{D} \mathcal{D}^{*}+\tilde{m}^{2}}
\end{array}\right)
$$

Par conséquent, comme nous le montrons maintenant, la phase de diffusion pour l'opérateur de Dirac avec potentiel magnétique est liée à celle associée à son carré, dont le comportement asymptotique est connu [15].

De façon analogue, en utilisant un théorème d'indice dû à N. V. Borisov, W. Müller et R. Schrader [3], nous pourrons aborder l'étude de l'opérateur de Hodge-Dirac agissant sur les formes différentielles, dont le carré est l'opérateur de Hodge-Laplace.

\subsection{Dirac avec champ magnétique}

L'opérateur de Dirac avec champ magnétique $H_{A}=c \alpha \cdot(\hbar \mathrm{D}-A)+m c^{2} \beta$ est un opérateur de Dirac supersymétrique. Il est de la forme (24) avec

$$
\mathcal{H}_{+}=\mathcal{H}_{-}=L^{2}\left(\mathbb{R}^{3}\right)^{2}, \quad \tilde{m}=m c^{2}, \quad \mathcal{D}^{*}=\mathcal{D}=c \sigma \cdot(\hbar \mathrm{D}-A(x)) .
$$

$\sigma_{1}, \sigma_{2}$ et $\sigma_{3}$ étant les $2 \times 2$-matrices de Pauli.

Pour démontrer le théorème 2.3 , nous montrons que la phase de diffusion pour le couple $\left(H_{A}, H_{0}\right)$ est directement liée à la phase de diffusion pour des opérateurs de Pauli.

Comme précédemment, nous posons $m=c=\hbar=1$. 
Asymptotique de la phase de diffusion à haute énergie pour l'opérateur de Dirac

LemMe 6.1. - On suppose que le champ magnétique $A$ vérifie $\left(H_{\delta}\right)$ avec $\delta>3$. Pour toute fonction $\phi \in C_{0}^{\infty}(] 0,+\infty[)$, on a

$$
\operatorname{Tr}\left(H_{A} \phi\left(H_{A}^{2}\right)-H_{0} \phi\left(H_{0}^{2}\right)\right)=0 .
$$

Preuve. - Dans $\mathcal{L}\left(L^{2}\left(\mathbb{R}^{3}\right)^{2} \oplus L^{2}\left(\mathbb{R}^{3}\right)^{2}\right), H_{A} \phi\left(H_{A}^{2}\right)$ s'exprime matriciellement par

$$
H_{A} \phi\left(H_{A}^{2}\right)=\left(\begin{array}{cc}
\phi\left(\mathcal{D}_{A}^{*} \mathcal{D}_{A}+1_{2}\right) & \mathcal{D}_{A}^{*} \phi\left(\mathcal{D}_{A} \mathcal{D}_{A}^{*}+1_{2}\right) \\
\mathcal{D}_{A} \phi\left(\mathcal{D}_{A}^{*} \mathcal{D}_{A}+1_{2}\right) & -\phi\left(\mathcal{D}_{A} \mathcal{D}_{A}^{*}+1_{2}\right)
\end{array}\right)
$$

(de même pour $H_{0} \phi\left(H_{0}^{2}\right)$ ). Or ici $\mathcal{D}_{A}^{*}=\mathcal{D}_{A}=\sigma \cdot(\mathrm{D}-A)$ et do' lc pour $L_{A}=\mathcal{D}_{A}^{2}+1_{2}$, on a

$$
\left.\operatorname{Tr}\left(H_{A} \phi\left(H_{A}^{2}\right)-H_{0} \phi\left(H_{0}^{2}\right)\right)=\operatorname{Tr}\left(\phi\left(L_{A}\right)-\phi\left(L_{0}\right)\right)-\operatorname{Tr}\left(\phi\left(L_{A}\right)-\epsilon_{A} L_{0}\right)\right)
$$

ce qui est évidemment nul. D'où le lemme.

En appliquant la formule de séparation des énergies (3), et le lemme précédent à $\phi(\lambda)=(\sqrt{\lambda})^{-1} f( \pm \sqrt{\lambda})$, on obtient la proposition suivante.

Proposition 6.2.- On suppose que le champ magnétique $A$ vérifie $\left(H_{\delta}\right)$ avec $\delta>3$. Pour toute fonction $f \in C_{0}^{\infty}(\mathbb{R} \backslash\{0\})$, on a :

$$
\begin{aligned}
& \operatorname{Tr}\left(f\left(H_{A}\right)-f\left(H_{0}\right)\right)= \\
& \quad=\frac{1}{2} \operatorname{Tr}\left(f\left(\left|H_{A}\right|\right)-f\left(\left|H_{0}\right|\right)\right)+\frac{1}{2} \operatorname{Tr}\left(f\left(-\left|H_{A}\right|\right)-f\left(-\left|H_{0}\right|\right)\right) .
\end{aligned}
$$

Dans le corollaire suivant, nous en déduisons une relation entre la phase de diffusion pour la paire $\left(H_{A}, H_{0}\right)$ et celle pour la paire $\left(h_{A}, h_{0}\right)$ d'opérateurs de Pauli

$$
h_{A} \stackrel{\text { def }}{=} \frac{(\sigma \cdot(\mathrm{D}-A))^{2}}{2}, \quad h_{0} \stackrel{\text { def }}{=}-\frac{\Delta}{2} 1_{2} .
$$


Corollaire 6.3.- On suppose que $A$ vérifie $\left(H_{\delta}\right)$ avec $\delta>3$. Les phases de diffusion $s_{D}^{A}$ et $s_{P}^{A}$, associées respectivement aux couples $\left(H_{A}, H_{0}\right)$ et $\left(h_{A}, h_{0}\right)$ sont définies et vérifient :

$$
s_{D}^{A}(\lambda)= \pm s_{P}^{A}\left(\frac{\lambda^{2}-1}{2}\right), \quad \pm \lambda>1
$$

(on a pris $s_{D}^{A}$ nulle sur $]-1,1$ [ et $s_{P}^{A}$ nulle sur $]-\infty, 0[$ ).

Preuve. - D'après la formule de Krein (1) appliquée à la phase $s_{D}$ pour la paire $\left(H_{A}, H_{0}\right)$ et à la phase $s_{L}$ pour la paire $\left(H_{A}^{2}, H_{0}^{2}\right)$, la proposition précédente donne que pour toute fonction $f \in C_{0}^{\infty}(\mathbb{R} \backslash\{0\})$, on a

$$
\int_{-\infty}^{+\infty} s_{D}(\mu) f^{\prime}(\mu) \mathrm{d} \mu=\frac{1}{2} \int_{0}^{+\infty} s:\left(\mu^{2}\right) f^{\prime}(\mu) \mathrm{d} \mu-\frac{1}{2} \int_{-\infty}^{0} s_{L}\left(\mu^{2}\right) f^{\prime}(\mu) \mathrm{d} \mu .
$$

Les fonctions $s_{D}$ et $s_{L}$ étant rés lières respectivement sur ] $-\infty,-1[\cup$ ] $1,+\infty[$ et $] 1,+\infty\left[\right.$, on en déd it $_{\text {it }}$ que pour $\pm \mu>1$ on a

$$
s_{D}(\mu)= \pm \frac{1}{2} s_{L}\left(\mu^{2}\right) .
$$

Pour conclure, il reste à constater que dans $\mathcal{L}\left(L^{2}\left(\mathbb{R}^{3}\right)^{2} \oplus L^{2}\left(\mathbb{R}^{3}\right)^{2}\right)$, on a

$$
H_{A}^{2}=\left(\begin{array}{cc}
2 h_{A}+1 & 0 \\
0 & 2 h_{A}+1
\end{array}\right)
$$

et donc que $s_{L}(\lambda)=2 s_{P}((\lambda-1) / 2)$.

Pour obtenir l'asymptotique complète du théorème 2.3 , on peut alors utiliser les travaux de D. Robert [15] où l'existence d'un développement asymptotique complet de la phase de diffusion est établie pour des perturbations du laplacien, en particulier pour $s_{P}^{A}$.

\subsection{Opérateur de Hodge-Dirac}

Un second exemple standard d'opérateur de Dirac supersymétrique est l'opérateur de Hodge-Dirac agissant sur l'espace des formes différentielles, dont le carré est l'opérateur de Hodge-Laplace.

Ces opérateurs sont définis sur $M$, une variété Riemannienne complète, de la façon suivante. Soit $d$ la différentielle extérieure et $\delta$ la codifférentielle 
Asymptotique de la phase de diffusion à haute énergie pour l'opérateur de Dirac

(c.-à-d. l'adjoint de $\mathrm{d}$ par la dualité de Hodge). Alors $Q=\mathrm{d}+\delta$ est un opérateur autoadjoint sur $\mathcal{H}$, l'espace des formes différentielles de carré intégrable. Si $P$ désigne l'opérateur qui à une $p$-forme associe l'entier $p$, alors $\tau=(-1)^{P}$ est une involution unitaire autoadjointe telle que $\tau Q+Q \tau=0$. Ainsi, $Q$ est une supercharge par rapport à $\tau, Q^{2}=$ $(\mathrm{d}+\delta)^{2}=\mathrm{d} \delta+\delta \mathrm{d}$ est un hamiltonien supersymétrique (c'est l'opérateur de Hodge-Laplace), et $H=Q+(-1)^{P} m$ (avec $m>0$ ) est un opérateur de Dirac supersymétrique, appelé opérateur de Hodge-Dirac. Les sous-espaces bosonique $\mathcal{H}_{+}$et fermionique $\mathcal{H}_{-}$sont respectivement l'ensemble des $2 p$ formes et des $(2 p+1)$-formes.

L'étude de la diffusion pour ces opérateurs s'effectue en les comparant aux opérateurs de Hodge-Dirac, $H_{0}$ et de Hodge-Laplace, $Q_{0}^{2}$ agissant sur $\mathcal{H}_{0}$, l'espace des formes différentielles de carré intégrable sur $\mathbb{R}^{n}$ muni de la métrique euclidienne $g_{0}$.

Nous considérons les variétés asymptotiquement plates de dimension $n$. Il s'agit de variétés riemanniennes non compactes $(M, g)$, dont les bouts infinis sont difféomorphes, par $\varphi$, au complémentaire d'une boule de $\mathbb{R}^{n}$, et $\left(\varphi_{*} g-g_{0}\right)$ vérifie $\left(\mathrm{H}_{\delta}\right)$ (cf. [3, chap. 5] pour plus de détails).

Les phases de diffusion, pour les couples $\left(H, H_{0}\right)$ et $\left(Q^{2}, Q_{0}^{2}\right)$, seront définies pour $\delta>n$.

La différence avec l'opérateur de Dirac relativiste (avec champ magnétique) est que nous n'aurons plus nécessairement le lemme 6.1. Celui-ci sera remplacé par une relation faisant intervenir la notion d'indice relatif (du type Witten) introduit par N. V. Borisov, W. Müller et R. Schrader [3]. On prévoit alors d'obtenir la relation suivante entre $s_{D}$, la phase de diffusion pour le couple $\left(H, H_{0}\right), s_{L}$, la phase de diffusion pour le couple $\left(Q^{2}, Q_{0}^{2}\right)$ et ind, l'indice relatif

$$
s_{D}(\lambda)= \pm \frac{1}{2} s_{L}\left(\lambda^{2}-m^{2}\right)-\frac{1}{2} \text { ind }, \quad \pm \lambda>m .
$$

Le développement asymptotique de $s_{D}$ pour $\lambda \rightarrow \pm \infty$ se déduira alors du développement asymptotique de $s_{L}$ pour $\lambda \rightarrow+\infty$.

L'élaboration de ces résultats est en cours. Pour la variété triviale $M=\mathbb{R}^{n}$, nous démontrons dans [4] que l'indice existe et est nul. Ainsi, grâce au développement asymptotique de $s_{L}$ donné par $\mathrm{D}$. Robert [15]; la relation (26), nous permet de déduire un développement asymptotique complet de $s_{D}$, pour une métrique asymptotiquement plate, dont les géodésiques ne sont pas captées. 
Pour une variété plate à l'infini, N. V. Borisov, W. Müller et R. Schrader [3] établissent que l'indice est nul pour $n$ impair et pour $n$ pair

$$
\text { ind }=\int_{M} \mathcal{R}(x)=\chi(M),
$$

où $\mathcal{R}(x)$ est la forme de Chern-Gauss-Bonnet sur $(M, g)$ et $\chi(M)$ est la $L^{2}$-caractéristique d'Euler de $M$.

L'étude de l'indice relatif et l'existence d'un développement asymptotique complet pour $s_{L}$ sont à étendre au cas général des variétés asymptotiquement plates, afin d'obtenir un développement asymptotique complet de $s_{D}$.

\section{Remerciements}

Ce travail a été réalisé pendant la préparation de ma thèse au département de mathématiques de l'Université de Nantes. Pour avoir guidé mes premiers pas dans la recherche mathématique et pour ses conseils, je tiens à remercier D. Robert. Je remercie également les membres de l'équipe E.D.P. avec lesquels j'ai eu l'occasion d'échanger des idées durant ces trois années de thèse.

\section{Références}

[1] Balslev (E.) et Helffer (B.) .- Limiting Absorption Principle and Resonances for the Dirac Operator, Advances in Applied Mathematics 13 (1992), pp. 186-215.

[2] BIRMAN (M. S.) et KREIN (M. G.) .- On the theory of wave operators and scattering operators, Dokl. Akad. Nauk. S.S.S.R. 5 (1962), pp. 475-478.

[3] Borisov (N. V.), Müller (W.) et Schrader (R.) .- Relative Index Theorems and Supersymmetric Scattering Theory, Commun. Math. Phys. 114 (1988), pp. 475513.

[4] BRUneau (V.) .- Propriétés asymptotiques du spectre continu d'opérateurs de Dirac, Thèse, Université de Nantes (1995).

[5] BRuneau (V.) . - Sur le spectre continu de l'opérateur de Dirac : formule de Weyl, limite non relativiste, C.R. Acad. Sci. Paris, série I, 322 (1996), pp. 43-48.

[6] DAUge (M.) et ROBERT (D.) .- Weyl's formula for a class of pseudodifferential operators with negative order in $L^{2}\left(\mathbb{R}^{n}\right)$, Lecture Notes in Mathematics, Springer Berlin 1256 (1986), pp. 91-122.

[7] Egorov (Yu. V.) et Shubin (M. A.) . - Partial Differential Equations I, Encyclopaedia of Mathematical Sciences, Springer-Verlag 30 (1992).

[8] GoHBerg (I. C.) et KREJN (M. G.) .- Introduction à la théorie des opérateurs linéaires non autoadjoints, Dunod (1971). 
Asymptotique de la phase de diffusion à haute énergie pour l'opérateur de Dirac

[9] Helffer (B.) et ROBERT (D.) .- Comportement semi-classique du spectre des hamiltoniens quantiques elliptiques, Ann. Inst. Fourier, Grenoble 31, $n^{\circ} 3$ (1981), pp. 169-223.

[10] HelfFer (B.) et ROBert (D.) .- Calcul fonctionnel par la transformation de Mellin et opérateurs admissibles, J. Funct. Anal. 53 (1983), pp. 246-268.

[11] Helffer (B.) et Sjöstrand (J.) .- Opérateurs de Schrödinger avec champs magnétiques faibles et constants, Exposé $n^{\circ}$ XII, Séminaire E.D.P., février 1989, Ecole Polytechnique.

[12] Pladdy (C.), SaITo (Y.) et Umeda (T.) . - Asymptotic behavior of the resolvent of the Dirac Operator, Mathematical results in quantum mechanics (Blossin, 1993), pp. 45-54; Oper. Theory, Adv. Appl. Birkhäuser, Basel, 70 (1994).

[13] RoBert (D.) .- Autour de l'approximation semi-classique, P. M. Birkhäuser 68 (1987).

[14] ROBERT (D.) .- Asymptotique à grande énergie de la phase de diffusion pour un potentiel, Asymptotic Analysis 3 (1991), pp. 301-320.

[15] ROBERT (D.) .- Asymptotique de la phase de diffusion à haute énergie rour des perturbations du second ordre du Laplacien, Ann. Sci. E.N.S. 25 (1992), pp. 07-134.

[16] RoBert (D.) .- Relative Time-Delay for Perturbations of Elliptic Operi ors and Semiclassical Asymptotics, Journal of Functional Analysis 126, n⿳ 1 (1994 pp. 3682.

[17] Robert (D.) et Tamura (H.) .- Semiclassical Asymptotics for Local Spectral Densities and Time Delay Problems in Scattering Process, Journal of Functional Analysis 80, $\mathrm{n}^{\circ} 1$ (1988), pp. 124-147.

[18] Seeley (R. T.) .- Complex powers of an elliptic operator, Proc. Sympos. Pure Math., A.M.S. 10 (1967), pp. 288-307.

[19] Thaller (B.) . - The Dirac equation, Texts and Monographs in Physics, SpringerVerlag (1992).

[20] Yajima (K.) .- The quasi-classical approximation to Dirac equation, I, J. Fac. Sci. Univ. Tokyo 29 (1982), pp. 161-194.

[21] YAMADA (0.) .- On the principle of limiting absorption for the Dirac operators, Publ. Res. Inst. Math. Sci. Kyoto Univ. 8 (1972-73), pp. 557-577. 